\title{
A systematic screen for genes expressed in definitive endoderm by Serial Analysis of Gene Expression (SAGE) Juan Hou ${ }^{1}$, Anita M Charters ${ }^{2}$, Sam C Lee ${ }^{1}$, Yongjun Zhao ${ }^{2}$, Mona K Wu', Steven JM Jones ${ }^{2,3}$, Marco A Marra ${ }^{2,3}$ and Pamela A Hoodless*1,3,4
}

Address: ${ }^{1}$ Terry Fox Laboratory, British Columbia Cancer Agency, Vancouver, Canada, ${ }^{2}$ Michael Smith Genome Sciences Centre, British Columbia Cancer Agency, Vancouver, Canada, ${ }^{3}$ Department of Medical Genetics, University of British Columbia, Vancouver, Canada and ${ }^{4}$ Terry Fox Laboratory, British Columbia Cancer Agency, 675 West 10th Avenue, Vancouver, BC, V5Z 1L3, Canada

Email: Juan Hou - jhou@bccrc.ca; Anita M Charters - acharters@bcgsc.ca; Sam C Lee - slee@bccrc.ca; Yongjun Zhao - yzhao@bcgsc.ca; Mona KWu - mwu@bccrc.ca; Steven JM Jones - sjones@bcgsc.ca; Marco A Marra - mmarra@bcgsc.ca; Pamela A Hoodless* - hoodless@bccrc.ca

* Corresponding author

Published: 2 August 2007

BMC Developmental Biology 2007, 7:92 doi:10.1186/147/-2/3X-7-92

Received: 24 April 2007

Accepted: 2 August 2007

This article is available from: http://www.biomedcentral.com/I47I-2/3X/7/92

(C) 2007 Hou et al; licensee BioMed Central Ltd.

This is an Open Access article distributed under the terms of the Creative Commons Attribution License (http://creativecommons.org/licenses/by/2.0), which permits unrestricted use, distribution, and reproduction in any medium, provided the original work is properly cited.

\begin{abstract}
Background: The embryonic definitive endoderm (DE) gives rise to organs of the gastrointestinal and respiratory tract including the liver, pancreas and epithelia of the lung and colon. Understanding how DE progenitor cells generate these tissues is critical to understanding the cause of visceral organ disorders and cancers, and will ultimately lead to novel therapies including tissue and organ regeneration. However, investigation into the molecular mechanisms of DE differentiation has been hindered by the lack of early DE-specific markers.
\end{abstract}

Results: We describe the identification of novel as well as known genes that are expressed in DE using Serial Analysis of Gene Expression (SAGE). We generated and analyzed three longSAGE libraries from early DE of murine embryos: early whole definitive endoderm (0-6 somite stage), foregut (8-12 somite stage), and hindgut (8-12 somite stage). A list of candidate genes enriched for expression in endoderm was compiled through comparisons within these three endoderm libraries and against 133 mouse longSAGE libraries generated by the Mouse Atlas of Gene Expression Project encompassing multiple embryonic tissues and stages. Using whole mount in situ hybridization, we confirmed that $22 / 32(69 \%)$ genes showed previously uncharacterized expression in the DE. Importantly, two genes identified, Pyy and 573052IE/2Rik, showed exclusive DE expression at early stages of endoderm patterning.

Conclusion: The high efficiency of this endoderm screen indicates that our approach can be successfully used to analyze and validate the vast amount of data obtained by the Mouse Atlas of Gene Expression Project. Importantly, these novel early endoderm-expressing genes will be valuable for further investigation into the molecular mechanisms that regulate endoderm development.

\section{Background}

The definitive endoderm (DE) is a population of multipotent stem cells allocated as one of the primary germ lay- ers during gastrulation. Initially formed as an epithelial sheet of approximately 500-1000 cells around the distal cup of an E7.5 mouse embryo, the DE is rapidly organized 
into a tube that runs along the anterior-posterior axis of the embryo [1-3]. The DE gives rise to the major cell types of many internal organs, including the thyroid, thymus, lung, stomach, liver, pancreas, intestine and bladder. Most of these organs have secretory and/or absorptive functions and play important roles in controlling body metabolism. Interest in the endoderm has intensified recently because processes that govern early development of DE-derived tissues may be recapitulated during stem cell differentiation $[4,5]$, which could provide future therapies for diseased adult organs. Understanding how DEderived organs are specified, differentiate, proliferate, and undergo morphogenesis is key to understanding visceral organ disorders and tissue regeneration.

The last decade has yielded great insights into the molecular regulation of DE development [6]. In particular, pathways governing the initial formation of $\mathrm{DE}$, patterning of the foregut, and morphogenesis of foregut-derived organs such as the pancreas and liver, have begun to be deciphered. Many of the key genes involved in the initial formation of DE are evolutionarily conserved. They include Nodal and components of its signaling pathway, transcription factors of the mix-like paired homeodomain class, Forkhead domain factors, and Sox17 HMG domain proteins [7-11]. Studies of ventral foregut patterning suggest that endoderm patterning is controlled by soluble factors provided by an adjacent germ layer [12]. FGF4, which is expressed in the neighboring cardiac mesoderm, can induce the differentiation of ventral foregut endoderm in a concentration-dependent manner $[13,14]$. FGF2 and Activin, secreted by the notochord, lead to the expression of pancreatic markers by repressing expression of Shh in pancreatic endoderm [15-19]. However, the precise hierarchical relationships between these factors and their downstream targets are still largely unknown, and complete molecular hierarchies have not been obtained. In addition, midgut and hindgut development is largely unexplored.

Embryonic stem (ES) cells have attracted much attention as a possible source of cells for regenerative medicine. Directing differentiation efficiently into specific lineages at high purities from ES cells requires both optimal selective culture conditions and markers to guide and monitor the differentiation process. While several methods of differentiation of ES cells to hepatic and insulin-producing cells have been described, determining the precise identity of these cells is problematic due to a lack of suitable markers [20-23]. More recently, two groups achieved efficient differentiation of human and murine ES cells into DE by combining directive culture conditions (serum concentration reduction and Activin supplements) and FACS sorting using the cell surface marker, CXCR4 $[4,5,24]$. Although useful, CXCR4 is not an ideal marker for the DE as it is widely expressed in the gastulation stage mouse embryo (Table 1 and $[5,25]$ ). At present there is no DEspecific marker that can unequivocally identify this cell type.

In summary, one major hurdle in the analysis of early DE development in both the embryo and ES cells is the lack of both pan-endodermal and endodermal region-specific genetic markers, since the majority of DE markers are also expressed in the visceral endoderm and/or other germ layers. Devising screens to identify genes specifically expressed in DE will contribute to studies of DE development. Several groups have carried out screens for novel genes expressed in the endoderm of Xenopus and mouse embryos using microarray or cDNA hybridization [2529]. Despite the identification of several endoderm enriched genes, no novel DE specific genes were identified. As an alternative approach, we used Serial Analysis of Gene Expression (SAGE) to provide quantitative gene expression profiles. SAGE has been improved by the development of a longSAGE protocol, which generates tags that are $21 \mathrm{bp}$ long and provides enhanced efficiency and accuracy of tag-to-gene mapping [30-32]. Compared with microarrays, SAGE has the additional advantage that it permits the identification of novel transcripts. SAGE also has the added benefit that the data are digital and thus can be easily shared among investigators and compared across different experiments and tissues.

In this study, we generated and analyzed three mouse DE longSAGE libraries. A list of candidate genes enriched for expression in endoderm was compiled through comparisons within these three endoderm libraries and against 133 mouse longSAGE libraries representing multiple embryonic stages and tissues generated by the Mouse Atlas of Gene Expression Project [32,33]. Sixty nine percent of these candidate genes showed previously uncharacterized expression in restricted tissues, including DE, after further whole mount in situ hybridization validation. Importantly, two genes identified, $P y y$ and 5730521E12Rik, showed exclusive DE expression at early stages of endoderm patterning. The high efficiency of this screen suggests that our endoderm libraries and the SAGE library database are powerful resources to identify tissue specific genes. Furthermore, these new endoderm genes provide a valuable tool for further investigation into the molecular mechanisms regulating endoderm development.

\section{Results}

\section{Overview of the endoderm libraries}

Enriched definitive endoderm tissue was obtained by a combination of proteolysis and manual micro-dissection methods [14]. After removing the extra-embryonic region and digestion with trypsin, the DE was separated from 
Table I: Tag counts for endoderm and ectoderm genes in the endoderm and ectoderm SAGE libraries.

\begin{tabular}{|c|c|c|c|c|c|c|c|}
\hline gene symbol & tag sequence & $\begin{array}{c}\text { Early Endoderm } \\
(108579)\end{array}$ & $\begin{array}{l}\text { Foregut } \\
(102972)\end{array}$ & $\begin{array}{l}\text { Hindgut } \\
\text { (1 1 0529) }\end{array}$ & $\begin{array}{c}\text { Neural } \\
\text { tube }(97364)\end{array}$ & $\begin{array}{c}\text { Anterior } \\
\text { neuropore } \\
(103594)\end{array}$ & $\begin{array}{c}\text { Posterior } \\
\text { neuropore } \\
(102196)\end{array}$ \\
\hline
\end{tabular}

endoderm genes

\begin{tabular}{|c|c|c|c|c|c|c|c|}
\hline Cxcr4 & TGAATGAGTGTCTAGGC & 5 & I & 6 & 2 & 2 & 2 \\
\hline Ecad & TAATGTTGCTAGAGTGA & 9 & 9 & 8 & 0 & 0 & I \\
\hline Foxal & TTAACGACAAAAAAAAA & 5 & 4 & I & 0 & I & 0 \\
\hline Foxa2 & GTGAAATCCAGGTCTCG & 8 & 6 & 9 & I & 2 & 0 \\
\hline Foxa3 & CTGCTATGCACCAAGAT & 2 & I & 3 & 0 & 0 & 0 \\
\hline Gata4 & ССТGССССТССТССАСА & I & 2 & I & 0 & 0 & 0 \\
\hline Gata6 & TACACAATAATTTTTTTT & 3 & 6 & 3 & 0 & 0 & 0 \\
\hline Hhex & TATATAGCATTACTTCT & 2 & 4 & I & 0 & 0 & 0 \\
\hline Ihh & GGAGAATTTTGGGAATG & 2 & 0 & 4 & 0 & 0 & 0 \\
\hline Shh & TTCTTGGAAACCAAGAC & 11 & 10 & 7 & $\mathrm{I}$ & 0 & 0 \\
\hline Sox 17 & CGTGTTTTCTCAATCTT & 21 & 2 & 8 & 0 & 0 & 0 \\
\hline
\end{tabular}

ectoderm genes

\begin{tabular}{|c|c|c|c|c|c|c|c|}
\hline Fgfl5 & ACTTGTTTTCTACATTA & 0 & 0 & 0 & 1 & 4 & 2 \\
\hline Hes5 & TGGGAGAACACAGGCTG & 0 & 0 & 0 & I & 3 & 2 \\
\hline Ncad & TTAATATCTTTCGTTAT & 0 & 0 & I & 3 & 2 & 3 \\
\hline Pax6 & GATTTAAGAGTTTTATC & 0 & 0 & 0 & 4 & I & I \\
\hline Sox2 & TATATATTTGAACTAAT & I & 3 & I & 6 & 5 & 10 \\
\hline Sox3 & TACCTGCCACCTGGCGG & 0 & 0 & 0 & 3 & 4 & 3 \\
\hline Zic2 & TGATGTTTCAGTGCTTT & 0 & 0 & 0 & 6 & 4 & 4 \\
\hline Zic3 & AATAACAGAAAAGTGGA & 0 & 0 & 0 & I & I & 0 \\
\hline
\end{tabular}

The total number of tags in each library is bracketed in the column heading.

ectoderm and mesoderm (Figure 1A). Somite 0-6 endoderm pieces were pooled for the early whole endoderm library (SM108, Figure 1A). At this stage the newly formed endoderm has not yet been patterned, based on endoderm explant experiments [14,34]. Somite 8-12 endoderm was divided through the midgut into foregut and hindgut regions, and then pooled for the foregut and hindgut libraries respectively (SM107 and SM112, Figure 1B). By this stage endoderm patterning has initiated $[14,35]$. The notochordal plate at 0-6 somite stage and the notochord at 8-12 somite stage adjoin the DE and thus were included in the library [36].

A total of 322,208 tags were sequenced from these three longSAGE endoderm libraries [33]. Analysis of the three libraries revealed the expression of 54,093 different tagsequences (see Methods). There were 26,238 tagsequences present in the early endoderm library (SM108). Of these tag-sequences, $51 \%$ were unique to the early endoderm library as compared to the later endoderm libraries. Similarly, 25,097 and 25,509 tag-sequences were present in the foregut (SM107) and hindgut (SM112) libraries, respectively. In each of these libraries, approximately $50 \%$ of the tag-sequences were unique, compared to the other two endoderm libraries (Figure 2A). To determine which genes the tag-sequences represented, we first compared our tag-sequences to transcript databases (Refseq, MGC and Ensembl). Tag-sequences that did not correspond to annotated transcripts were then mapped to Ensembl gene units, which were extracted from the Ensembl database and include intronic regions and $1.0 \mathrm{~kb}$ upstream and downstream of annotated transcripts (Ensembl genes). Finally, tags were mapped to the mouse genome (UCSC). Of the combined 54,093 tag-sequences, $37 \%(19,782)$ mapped to known transcripts using the Refseq, MGC and Ensembl transcript databases, 12\% $(6,560)$ mapped to known genes using the Ensembl genes, implicating alternative splicing and alternative 3' UTRs of known genes, and 20\% $(10,954)$ mapped to the mouse genome. The remaining $31 \%(16,797)$ of the tagsequences did not map to any of these databases (Figure 2B). Ninety percent of these unmapped tag-sequences were single tags, implying that many may have been generated by sequencing, PCR, or other errors. We have previously shown that many of these tag-sequences can be mapped by allowing a one-basepair mismatch, insertion or deletion [33]. However, some of these tag-sequences likely represent valid, novel transcripts, since 44 unmapped tag-sequences expressed in the endoderm were found at a level of at least 4 tags. For example, these 44 tag-sequences may span an unknown splice junction [37]. To simplify the analysis and validation in this study, we 


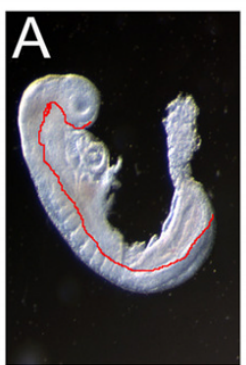

Somite 10
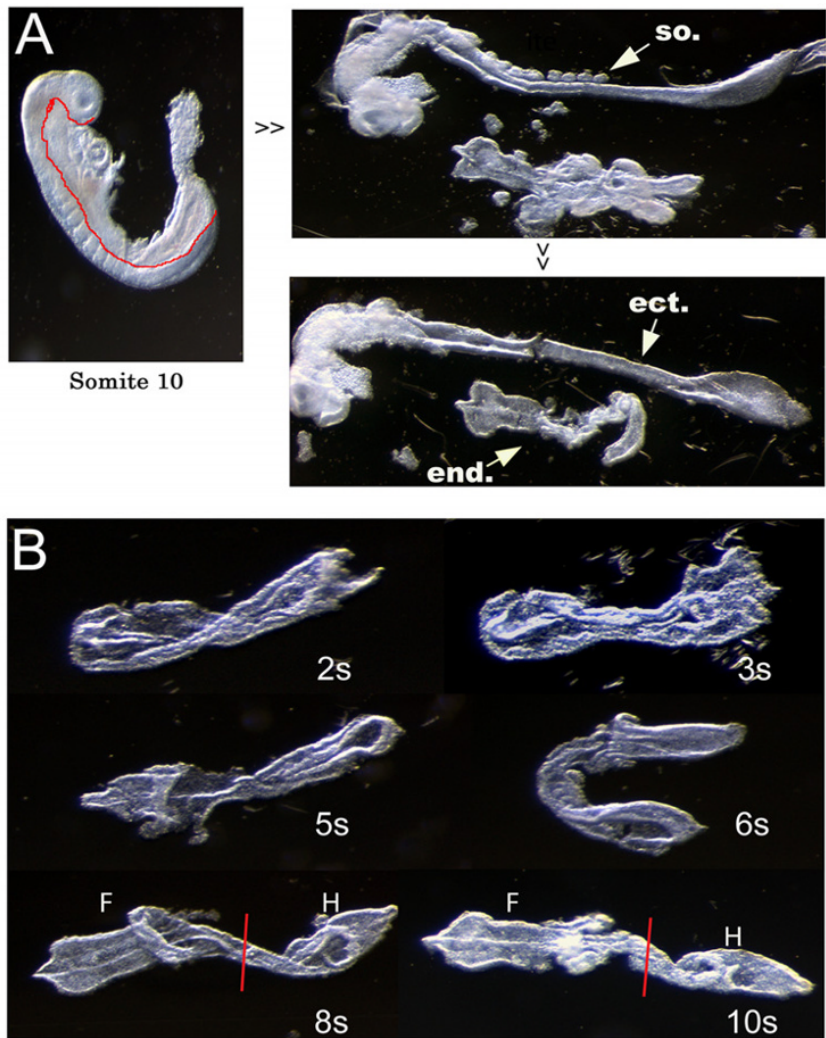

Figure I

Collection of definitive endoderm from E8.0-8.5 mouse embryo. (A) Dissection procedure and germ layer separation process. After trypsin treatment, ectoderm and endoderm can be separated (indicated by the red line). After the somites and mesoderm were removed, enriched ectoderm and endoderm can be obtained. (B) Photographs of the intact dissected endoderm at the indicated somite stage. Somite 0 6 endoderm pieces were pooled for the early whole endoderm library. Somite 8- 12 endodermal portions were separated into foregut and hindgut portions (indicated by the red line). So: somite; end: endoderm; ect: ectoderm; F: foregut; $\mathrm{H}$ : hindgut.

focused on tag-sequences that unambiguously mapped to the most 3' position (position number +1 ) and the sense strand of the Refseq database (refer to Methods); 7,084 tag-sequences (13\%) met these criteria.

To assess the quality of our endoderm libraries, we searched for genes known to be expressed in the endoderm (Cxcr4, Ecad, Foxa1-3, Gata4, Gata6, Hhex, Ihh, Shh, and Sox17) and ectoderm (Fgf15, Hes5, Ncad, Pax6, Sox2, Sox3, Zic2, Zic3) (Table 1). Since we also generated 3 ectoderm libraries from early somite stage mouse embryos, we evaluated the integrity of the libraries by comparing gene expression levels in the endoderm and ectoderm libraries. Significantly, all of these endoderm genes were present in

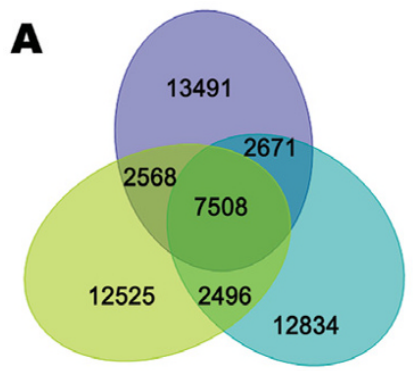

Whole early endoderm, 0-6 somite

Foregut, 8-12 somite

Hindgut, 8-12 somite

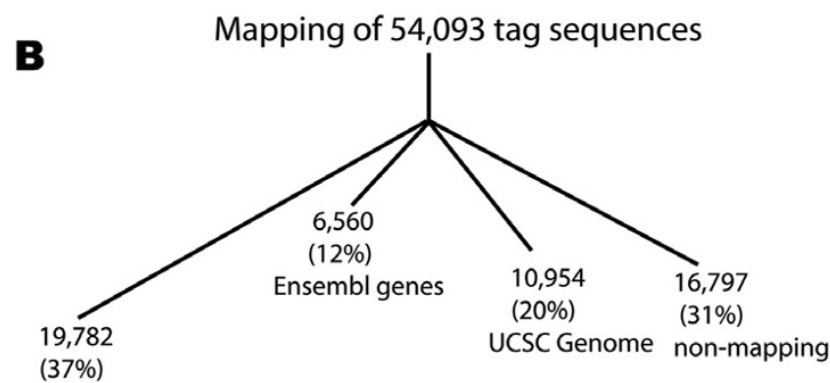

Refseq, MGC

Ensembl transcripts

\section{Figure 2}

Overview of the endoderm SAGE libraries. (A) Venn diagram summarizing the number of unique and common tagsequences in the three endoderm longSAGE libraries. (B) Summary of tag-to-gene mapping efficiencies. Additional details are in text.

our endoderm libraries and excluded or present at low levels in the ectoderm libraries. The exception is Cxcr4, which although used as a DE marker, was expressed in both endoderm and ectoderm, reaffirming it as widely expressed [25]. Similarly, Sox2 is expressed in both ectoderm and endoderm libraries corresponding to published expression patterns [38]. All of the other ectoderm genes present in our ectoderm libraries were excluded or present at low level in the endoderm libraries. Overall, the expression patterns observed in our libraries supports known expression data for these genes, indicating that the libraries are representative of endoderm and ectoderm transcription.

\section{Identification of foregut-specific genes}

To identify genes that were specifically expressed in the foregut or the hindgut, a cross-comparison between the two libraries (SM107 and SM112, respectively) was performed. An initial list of genes was made by selecting tagsequences that were present at counts $\geq 4$ for transcription factors (TFs) and signaling pathway components (SPCs), and counts $\geq 7$ for other genes, in either the foregut or hindgut library. This threshold allowed us to identify the 
top 25 most highly expressed tag-sequences present exclusively in the foregut library and the top 20 most highly expressed tag-sequences present exclusively in the hindgut library, which was a tractable number for further validation [see Additional file 1]. By screening with both semiquantitative RT-PCR and quantitative RT-PCR, 14 of the 45 genes were shown to exhibit differential expression between the foregut and hindgut. Whole mount in situ hybridization was performed on these 14 genes. Six of these genes showed a ubiquitous expression pattern, making it difficult to determine whether there was differential expression within the DE. However, 8 genes did exhibit differential expression levels between the foregut and hindgut (Figure 3). Seven of these genes, Trh, Otx2, Prrx2, Tbx1, Cyp26a1, Hoxb6, and $C d x 1$ were expressed in other tissues as well as endoderm at the early somite stage. Significantly, one of the genes, Pyy, was exclusively expressed in the foregut endoderm.

\section{Expression of Pyy in the early mouse embryo}

$P y y$ is known to be highly expressed in pancreatic islets and endocrine L cells of the lower gastrointestinal tract

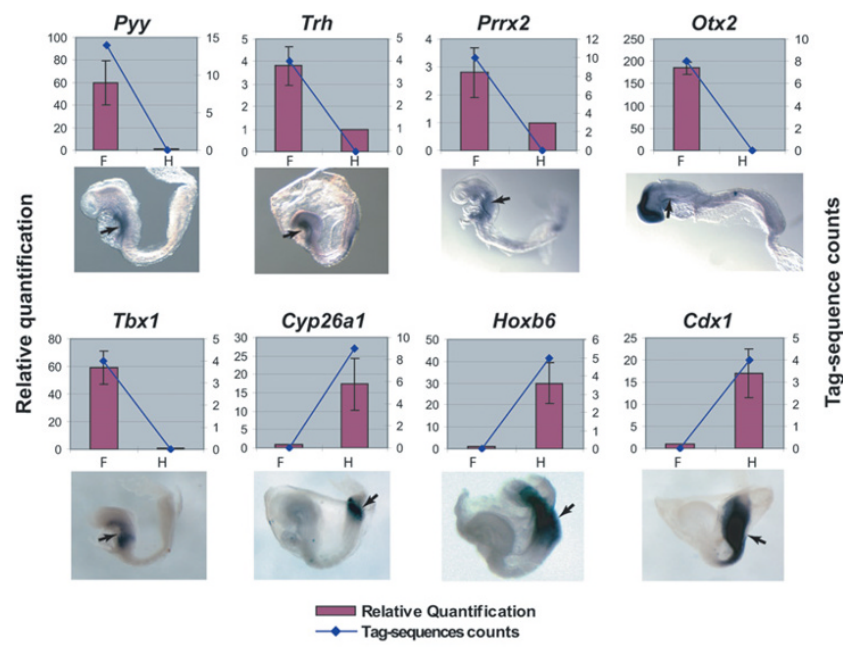

Figure 3

Correlation of the expression validation of 8 genes from the first list between RT-qPCR, whole mount in situ hybridization and SAGE. For each gene, the upper panel shows the comparison of expression level using RT-qPCR and SAGE (Left scale: relative quantification indicated by the bars; Right scale: raw tag-sequence counts indicated by the line. F: foregut; $\mathrm{H}$ : hindgut). The lower panel shows the expression pattern detected by whole mount in situ hybridization. For all embryos, anterior is to the left and posterior is to the right. The RT-qPCR, whole mount in situ hybridization and SAGE validation results were well correlated. PYY, Trh, Prrx2, Otx2 and $T b x I$ are highly expressed in the foregut (indicated by arrow). Conversely, Cyp26al, Hoxb6 and $C d x I$ are highly expressed in the hindgut (indicated by arrow).
[39], but its early embryonic expression pattern has not been described. Due to the exclusive expression of Pyy in the DE at early somite stages from our analysis, we further examined Pyy expression pattern during early mouse embryogenesis. Whole mount in situ hybridization was performed on embryos collected from E6.0 to E9.5 stages (Figure 4). Interestingly, Pyy was expressed in small lateral regions of the foregut $\mathrm{DE}$ as early as the 2 somite stage (Figure 4A, 4B). At the 4 somite stage, the expression domains in the lateral region were expanded and a second expression domain in the medial ventral foregut was observed (Figure 4C, 4D). Subsequently, the lateral expression domains expanded and extended anteriorly to
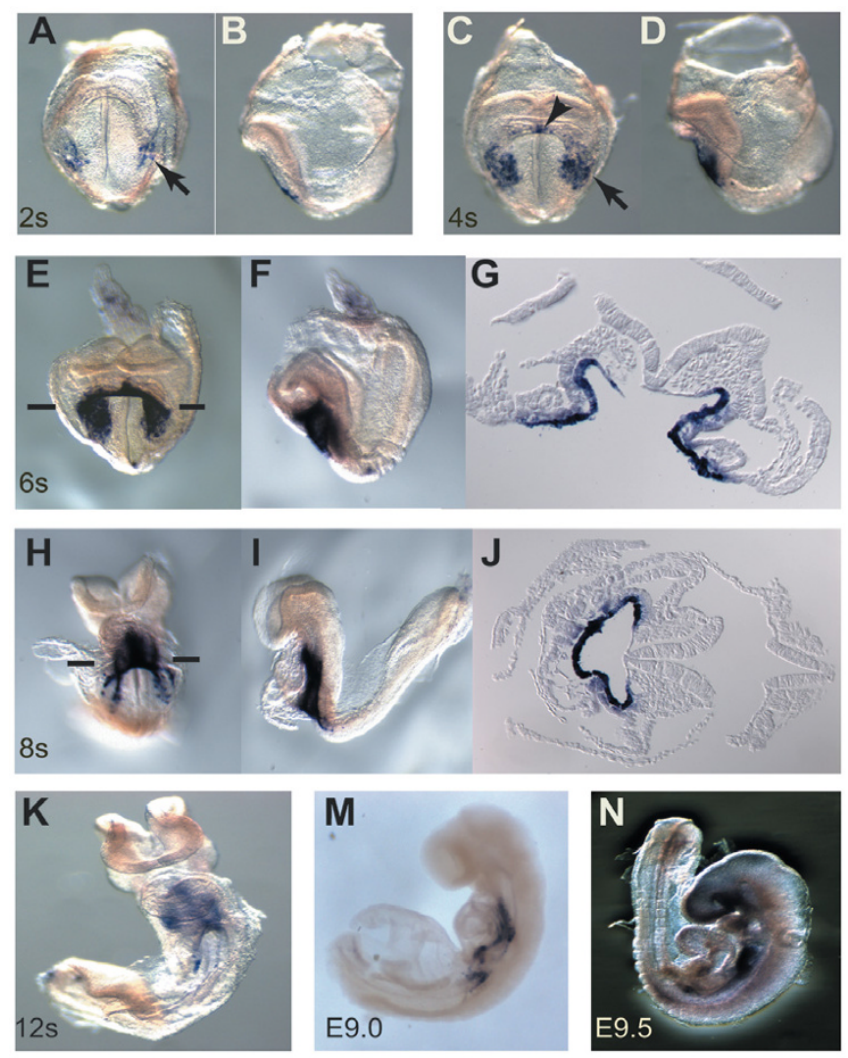

\section{Figure 4}

Expression of Pyy in the early developing mouse embryo. (A, B) Pyy expression is seen in small lateral region of the DE at as early as 2 somite stage (indicated by arrow). (C, D) At the 4 somite stage, the expression domains in the lateral region are expanded, and the second expression domain which is in the medial ventral foregut can to be observed (arrowhead). (E-J) The lateral expression domains expanded and extended anteriorly to the medial ventral foregut. Strong expression was observed in the lateral and ventral foregut in the 6-8 somite stages. Representative sections are shown in the right panel. (K-N) In the early organogenesis stage, the Pyy expression remained in the posterior foregut extending to the midgut junction. 
the medial ventral foregut, so that strong expression was observed in the lateral and ventral foregut at the 6-8 somite stages (Figure 4E-J). Interestingly, the expression was restricted to the posterior half of the foregut and never observed in the anterior half of the foregut pocket. At early organogenesis stage, $P y y$ expression remained in the posterior foregut extending to the midgut junction (Figure $4 \mathrm{~K}-\mathrm{N}$ ). Thus, $P y y$ is expressed earlier than previously reported and demonstrates a dynamic expression pattern in the early DE.

\section{Identification of novel genes expressed in the DE}

In addition to identifying foregut- and hindgut-enriched DE markers, we wanted to identify additional novel genes with distinct expression patterns in the endoderm to facilitate DE patterning studies. Thus, to increase the efficiency of identification of novel endoderm genes, we chose to exploit the Mouse Atlas of Gene Expression Project database, which contained 133 libraries from different tissues and stages of development. We reasoned that if a gene was ubiquitously expressed, it would be present in most of the libraries. Conversely, if the expression of a gene were restricted to a specific cell-type, it would be present only in a specific subset of libraries. Indeed, by examining the expression patterns of our original list (foregut vs hindgut) of 45 tag-sequences in 133 longSAGE libraries generated by the Mouse Atlas Project, we discovered 9 genes that exhibited high tissue-specificity since they were present in only a few libraries (Figure 5). Interestingly, 8 of the 9 genes demonstrating a tissue-restricted expression pattern matched the endoderm genes identified in our in situ hybridization analysis (Figure 3 ). This suggests that in the context of looking for specificity of gene expression, the SAGE data is an excellent tool for identifying genes with tissue restricted expression.

To identify genes expressed in the $\mathrm{DE}$, a second list was generated using tag-sequences present in the three endoderm libraries $(7,084$ tag sequences which were unambiguously mapped to the most 3 ' position and the sense strand of the Refseq database). We considered two factors, the total number of Mouse Atlas SAGE libraries in which a tag-sequence was present (L), and the total number of times that a tag-sequence was found in the three pooled endoderm libraries $(\mathrm{T})$. We rationalized that higher $\mathrm{T}$ values and lower $\mathrm{L}$ values and thus higher $\mathrm{T} / \mathrm{L}$ ratio would correspond to the degree of the endoderm-enrichment. We compiled a list consisting of tag-sequences with $\mathrm{T}>4$ and $\mathrm{L}<58$ and calculated the $\mathrm{T} / \mathrm{L}$ ratio [see Additional file $2]$. We removed the tag sequences whose $\mathrm{T} / \mathrm{L}$ ratio was less than 0.21 to create a second list consisting of 60 genes. Confirming the effectiveness of these criteria, 6 out of the 60 genes were present in and validated by our first list, and 24 out of the 60 genes were previously shown to be expressed in endoderm, either with or without expression

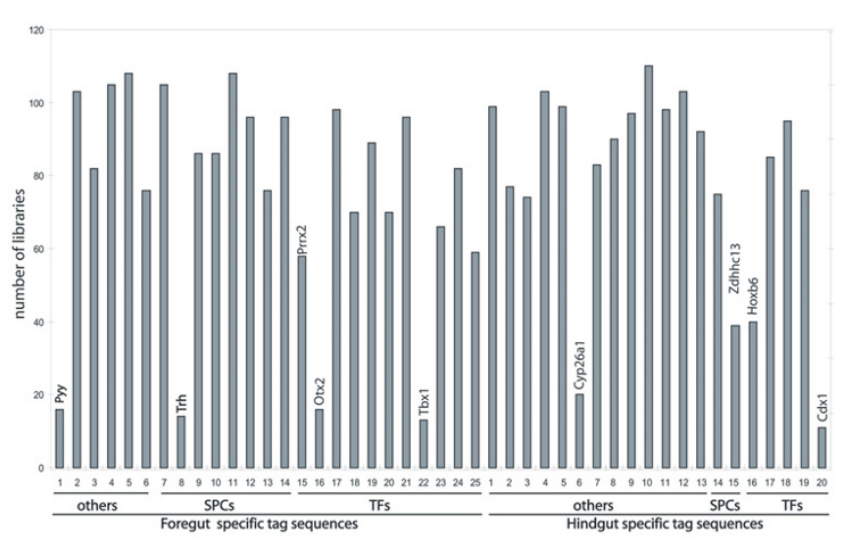

\section{Figure 5}

Expression of genes from the first candidate list within 133 mouse atlas SAGE libraries. Numbers on the $\mathrm{X}$-axis depict each tag-sequence in the first candidate list, and the $Y$-axis depicts the number of the libraries in which a specific tagsequence is present. Thus a low bar reflects high tissue specificity, and vice versa. The 8 genes from the Ist list exhibiting differential expression between foregut and hindgut by RTqPCR as well as whole mount in situ hybridization have significantly lower bars. Names of these genes are provided on top of their respective bars. SPCs: Signaling Pathway Components; TFs: Transcription Factors.

in other germ layer tissues, including Sox17, Foxa1-3, Ihh and Shh [see Additional file 2].

Of the remaining 30 genes, we successfully examined the expression of 26 genes using whole mount in situ hybridization. 21 of the 26 genes showed tissue-restricted expression patterns (Figure 6, 7 and Table 2), while the remaining 5 genes showed ubiquitous expression at E8.5. Including the 6 candidate genes validated from the first list, the efficiency of our new screen for novel genes with tissue-restricted expression patterns was $84 \%$ (27/32). Interestingly, we found the majority of genes identified were not only expressed in the definitive endoderm, but also in other tissues such as yolk sac, ectoderm and mesoderm. We classified the 27 genes exhibiting tissuerestricted expression into five categories, based on their expression patterns (Table 2). The first group includes two genes, 5730521E12Rik and $P y y$, which were expressed exclusively in the DE. We described the Pyy expression pattern in the early mouse embryo above and, the 5730521E12Rik expression pattern is described below. Group 2 included genes that were expressed in the definitive endoderm and yolk sac endoderm, which support the functional similarity between these two lineages [6]. Group 3 contained genes that were expressed in the DE, yolk sac and another germ layer with a tissue-restricted pattern, and Group 4 contained genes that were not expressed in yolk sac and heart, but expressed in all 3 germ 
layers. The genes in Group 5 were expressed in yolk sac endoderm at high levels, without obvious expression within the DE. The tags for these genes may be included in our libraries due to yolk sac endoderm contamination, which is difficult to avoid when collecting the DE tissue. Alternatively, these genes may be expressed in the DE at low levels but their expression in DE could be under-estimated by in situ hybridization due to very high levels in yolk sac endoderm. Thus most of the genes selected by our criteria for in situ hybridization showed complex tissuerestricted expression patterns in the early embryo, including the DE. Overall, these results indicate our approach was successful in the identification of novel markers of endoderm expression.

\section{Expression of 573052 I E I 2Rik in early mouse embryo} In addition to $P y y, 5730521 E 12 R i k$ exhibited exclusive expression in the DE at early somite stages in our analysis. We further examined the expression pattern of 5730521E12Rik during early mouse embryogenesis. Interestingly, 5730521E12Rik was first expressed in a few cells at E7.25 in the endoderm at the posterior region adjacent to the embryonic-extraembryonic junction (Figure 8A). At the late head-fold stage, 5730521E12Rik expression has expanded in the lateral region of the endoderm on the posterior side (Figure $8 \mathrm{~B}$ ). As development proceeds, the bilateral expression domains extended anteriorly and medially and began to focus in the midgut region at early somite stages (Figure 8C-F). By E9.0 strong expression was observed in the midgut (Figure 8G). At E9.5, 5730521E12Rik expression was still maintained in the midgut region with the expression level decreased (Figure 8H). Thus 5730521E12Rik expression was specific to the midgut during the gastrulation and early organogenesis stages in the mouse embryo.

\section{Discussion}

Formation, specification and patterning of the definitive endoderm are poorly understood in the embryo compared to other germ layers. Due to a lack of exploratory tools to aid these studies, interest in the identification of novel endoderm genes is growing. The recent enthusiasm for stem cell differentiation methodologies and the clinical potential for these cells have heightened the need for better tools and a further understanding of normal embryonic development. Several groups have undertaken largescale screens to identify novel genes that may be informative for developmental processes. In particular, in situ hybridization has been used to identify novel genes with unique expression patterns at mid-gestation (E9.5) [29]. However, while in situ hybridization is considered to be the ultimate and proven method to validate tissue-specific genes, obtaining embryos for in situ hybridization at appropriate stages is more costly and time-consuming in mouse than in chicken, frog or fish. Thus ensuring high efficiency in the screening for tissue-specific genes during mouse development is an important consideration.

To identify novel definitive endoderm specific genes, we used a longSAGE approach. We were able to enhance our screening efficiency since the endoderm longSAGE libraries were generated from enriched DE tissues at early stages of DE formation (E8.0-E8.5) that were obtained by a combination of proteolytic and manual micro-dissection methods. In addition, pre-selection of candidate genes by comparisons with 133 SAGE libraries from various tissues allowed us to eliminate $95 \%$ of the widely expressing genes from our endoderm libraries. Overall, the efficiency of our screen for genes with DE expression was 69\% (22/ 32 , not including Group 5 genes which are highly expressed in the yolk sac). By including genes expressed in the yolk sac visceral endoderm, we observed $84 \%(27 / 32)$ of genes identified with endoderm expression. Significantly, two of these genes, Pyy and the Riken gene, $5730521 E 12 R i k$, were exclusively expressed in the DE lineage at early organogenesis stages of development.

Previous studies focusing on screening for novel endoderm genes have used cDNA cloning or microarray analysis [25-29,40]. Sousa-Nunes et al. identified 29/160 (18\%) genes with restricted expression patterns from E7.5 mouse endoderm cDNA libraries using non-redundant sequence-based selection and in situ hybridization, but not all of these genes were endoderm enriched in their expression $[40,41]$. Sherwood et al. recently used fluorescent activated cell sorting to isolate definitive and visceral endoderm cell populations for microarray analysis [25]. They identified 18 out of 27 (67\%) novel genes whose expression was enriched in endoderm. They defined a pan-endodermal signature composed of 22 novel and known genes that is preferentially expressed in definitive and visceral endoderm. Interestingly, neither study was able to identify novel genes that are expressed specifically in the DE.

The lack of DE specific genes may be due to sensitivity and depth of screening. Furthermore, the high functional similarity between visceral and definitive endoderm suggests that these tissues have highly related transcriptomes [25]. Several genes in our study were found to be expressed in both visceral and definitive endoderm, supporting the similarity of the two tissues. It is likely that some endoderm-specific or enriched genes were excluded from the gene list determined by our selection criteria. Our SAGE sampling depth ( 100,000 tags per library) yields genedetection sensitivity approximately equivalent to that of fluorescence-based microarray approaches [42], and is thus sufficient for detection of abundant and moderately abundant transcripts but is likely insufficient for reliable detection of rare transcripts. Several previously known 
Table 2: Tissue restricted expression of the genes isolated by whole mount in situ hybridization.

\begin{tabular}{|c|c|c|c|c|c|c|c|}
\hline Gene symbol & Gene name & $\mathbf{L}$ & $\mathbf{F}$ & $\mathbf{E}$ & $\mathbf{H}$ & $\mathbf{T}$ & Expression pattern in E8.0-8.5 \\
\hline \multicolumn{8}{|c|}{ Group I: Genes expressed in definitive endoderm only } \\
\hline *Pyy & peptide $Y Y$ & 19 & 14 & 9 & 0 & 23 & foregut \\
\hline 573052 IEI2Rik & 573052 IEI2Rik & 9 & 9 & 25 & 63 & 97 & midgut \\
\hline
\end{tabular}

\section{Group2: Genes expressed in both definitive and yolk sac endoderm}

\begin{tabular}{clcccccl}
\hline Cldn9 & Claudin9 & 18 & 4 & 4 & 1 & 9 & definitive endoderm and yolk sac \\
Habp2 & Hyaluronic binding protein 2 & 25 & 0 & 2 & 4 & 6 & definitive endoderm and yolk sac \\
Spp2 & Secreted phosphoprotein2 & 16 & 1 & 7 & 2 & 10 & definitive endoderm and yolk sac \\
definitive endoderm and yolk sac
\end{tabular}

Group3: Genes expressed in definitive endoderm, yolk sac and other germ layers

\begin{tabular}{|c|c|c|c|c|c|c|c|}
\hline CpnI & $\begin{array}{l}\text { Carboxypeptidase } \mathrm{N}, \\
\text { polypeptide I }\end{array}$ & 28 & 2 & 5 & 6 & 13 & definitive endoderm, neural tube and yolk sac \\
\hline I 7000 I I HI 4Rik & I7000IIHI4Rik & 27 & 1 & 2 & 4 & 7 & definitive endoderm(weak), neural tube and yolk sac \\
\hline Mogat2 & $\begin{array}{l}\text { Monoacylglycerol O- } \\
\text { acyltransferase2 }\end{array}$ & 36 & 2 & II & 6 & 19 & $\begin{array}{c}\text { yolk sac endoderm, definitive endoderm, anterior ectoderm, and } \\
\text { somite }\end{array}$ \\
\hline Spink3 & $\begin{array}{l}\text { Serine peptidase inhibitor, Kazal } \\
\text { type } 3\end{array}$ & 38 & 44 & 109 & 57 & 210 & yolk sac endoderm, ectoderm and definitive endoderm \\
\hline Phlda2 & $\begin{array}{l}\text { Pleckstrin homology-like domain, } \\
\text { family A, member } 2\end{array}$ & 26 & 7 & 10 & 5 & 22 & $\begin{array}{c}\text { yolk sac endoderm, lateral plate mesoderm and ventral definitive } \\
\text { endoderm }\end{array}$ \\
\hline Trapla & Tumor rejection antigen $\mathrm{PIA}$ & 17 & $\mathrm{I}$ & 5 & 6 & 12 & definitive endoderm, yolk sac and midbrain and tailbud \\
\hline
\end{tabular}

Group4: Genes not expressed in yolk sac and heart, but expressed in other germ layers

\begin{tabular}{|c|c|c|c|c|c|c|c|}
\hline Gabpbl & GA repeat binding protein, beta I & 27 & 3 & 1 & 2 & 6 & $\begin{array}{l}\text { entire definitive endoderm, head and tailbud ectoderm and } \\
\text { mesoderm, but not expressed at heart and yolk sac }\end{array}$ \\
\hline$* C d x I$ & Caudal type homeo boxl & 13 & 0 & 17 & 4 & 21 & 3 germ layers of the posterior embryo \\
\hline *Trh & Thyrotropin releasing hormone & 18 & 4 & 9 & 0 & 13 & definitive endoderm, brain, midline \\
\hline *Cyp26al & $\begin{array}{l}\text { Cytochrome P450, family } 26 \text {, } \\
\text { subfamily a, polypeptide I }\end{array}$ & 24 & 0 & 5 & 9 & 14 & tailbud \\
\hline$* T b x I$ & T-boxl & 14 & 4 & I & 0 & 5 & foregut and mesoderm \\
\hline$* 0 t x 2$ & Orthodenticle homolog2 & 27 & 8 & 2 & 0 & 10 & brain, foregut \\
\hline $\operatorname{Arg} I$ & Arginase I, liver & 15 & 4 & 0 & 4 & 8 & $\begin{array}{c}3 \text { germ layers of the trunk, but not expressed at head, heart, tail } \\
\text { bud and yolk sac }\end{array}$ \\
\hline Gm784 & Gene model 784 & 37 & 6 & I & 5 & 12 & 3 germ layers, but not expressed at heart and yolk sac \\
\hline A230098A / 2Rik & A230098AI2Rik & 10 & 3 & I & I & 5 & 3 germ layers, but not expressed at heart and yolk sac \\
\hline Usp22 & Ubiquitin specific peptidase & 38 & 2 & 3 & 3 & 8 & 3 germ layers, but not expressed at heart and yolk sac \\
\hline
\end{tabular}

\section{Group5: Genes expressed in yolk sac endoderm only}

\begin{tabular}{ccccccccc}
\hline Tdh & L-threonine dehydrogenase & 19 & 1 & 7 & 11 & 19 & yolk sac endoderm \\
Lgals2 & Lectin, galactose-binding, soluble & 18 & 1 & 2 & 6 & 9 & yolk sac endoderm \\
Cubn & Cubilin (intrisic factor-cobalamin & 23 & 1 & 16 & 17 & 23 & yolk sac endoderm \\
receptor) & $\begin{array}{c}\text { Phospholipase A2, group I2B } \\
\text { ApolipoproteinC-2 }\end{array}$ & 16 & 2 & 2 & 1 & 5 & yolk sac endoderm \\
Apacgl2b & 17 & 3 & 13 & 7 & 23 & yolk sac endoderm \\
\hline
\end{tabular}

L: number of libraries which the 3' most tag sequence of each gene present in; F, E, H, T: raw counts of the $3^{\prime}$ most tag sequences for each gene in foregut library $(F)$, whole endoderm library $(E)$, hindgut library $(H)$ and in the three endoderm libraries $(T)$ respectively. *: genes validated in the first list. 


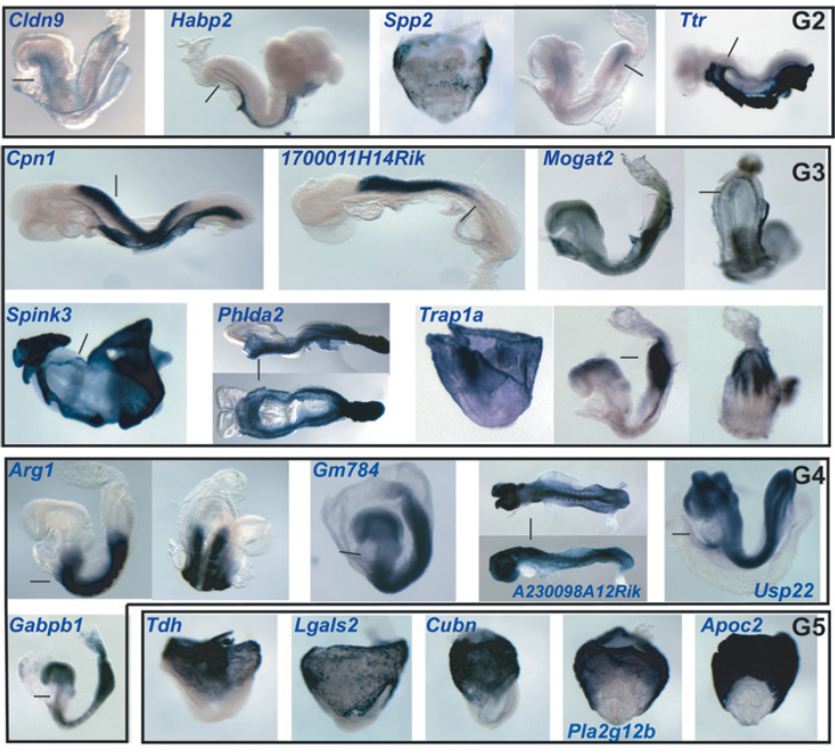

Figure 6

Whole mount in situ hybridization validation of the $2^{\text {nd }}$ candidate list, illustrating the complex expression patterns of endoderm genes. For explanation see text and Table 2 . The DE expressions of the genes in Groups 2-4 are shown further by histological sections in Figure 7.

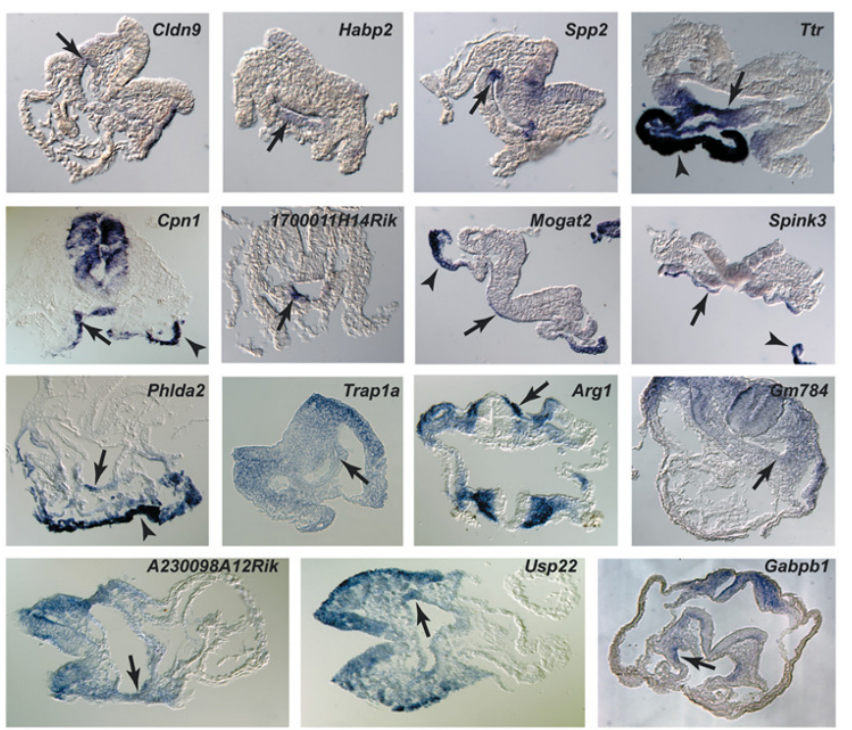

Figure 7

Histological sections through the embryos as indicated by the line in Figure 6, with arrows pointing to the DE staining. Arrowheads indicate staining in visceral endoderm.

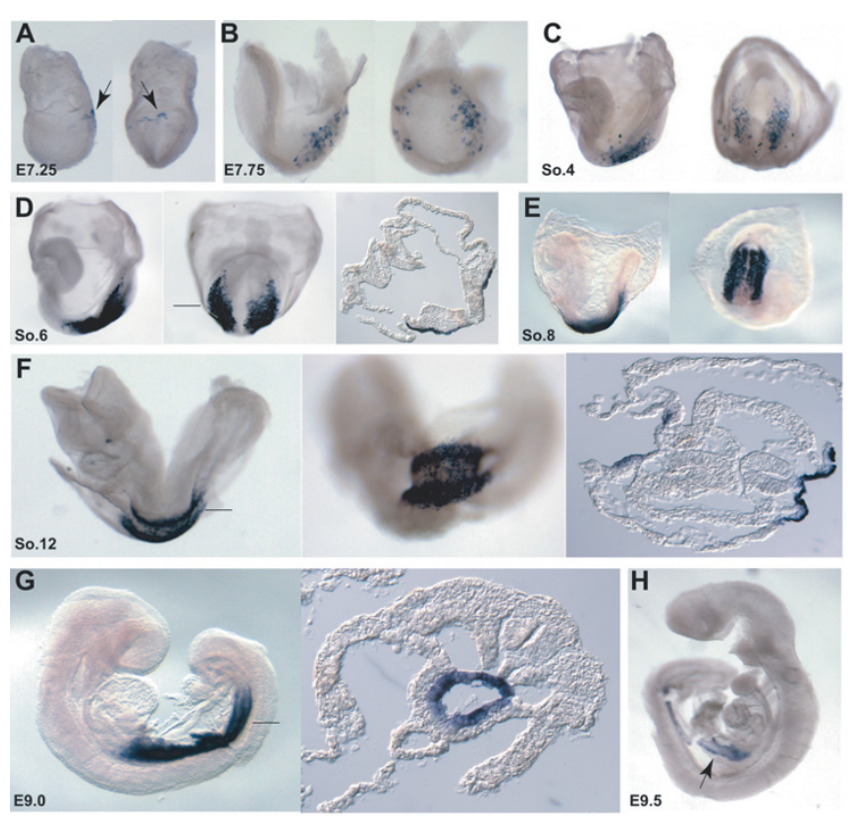

Figure 8

Expression of 573052 IEI 2 Rik in the early developing mouse embryo. 573052 IE I 2Rik expression from E6.5 to E9.5 in the mouse embryo was examined by whole mount in situ hybridization. The embryos at each represented stage are shown in lateral and posterior view (A-D) or lateral and ventral view $(E, F)$, except E9 and E9.5 (G, H). For the lateral view, the embryos are oriented so that the anterior is to left. The expression of 573052 IEI 2Rik is dynamic. Hybridization signals initiate at E7.25 in a few cells in the posterior of the embryos (arrowheads in A), then is continuously detected in broader bilateral domains of the middle-posterior of the embryos at head fold (B) and early somite (C-E) stages. At E8.5-E9 (E-G), 573052 IE I 2Rik expression level reaches the highest in the midgut. At E9.5, the signal is retained but is down-regulated $(\mathrm{H})$.

foregut or hindgut markers were not present in our list likely due to their low expression level and/or their expression being restricted to few cells within the endoderm. For example, Prox 1 is only expressed by the liver and pancreas progenitors beginning at the 7-8 somite stage [43]. Therefore, the Prox1 transcripts could be diluted by the total number of transcripts present in the foregut endoderm tissues, and thus not detected at our sequencing depth. With the advent of less expensive "next generation" sequencing this short-coming can be overcome by sequencing SAGE libraries to a greater depth. Furthermore, the foregut marker, Hhex, was missed in our analysis since it was expressed in 67 of our SAGE libraries, and thus did not fit our criteria for our validation lists (Table 1 and Additional file 2) [44]. Since many developmentally important genes are transcribed repeatedly and presumably function during multiple developmental processes, further refining of library and tissue choices for 
comparisons would be required to identify genes that are expressed in many stages of development and many tissues.

Pyy and 5730521E12Rik were identified to be exclusively expressed in the DE lineage at gastrulation and early organogenesis stages of development. Initially, $P y y$ is expressed at the early somite stage in the bilateral and medial regions of the foregut. Its early regionalized expression within DE reflects an early specification of cell fate along both the anterior-posterior and lateral-medial axis of the embryonic gut $[6,45]$. Subsequently, Pyy is expressed in the posterior foregut extending to the midgut junction, and at later stages (E14.5-adult) expression becomes restricted to the pancreas, stomach and intestine (data not shown). Interestingly, Tremblay et al. recently tracked progenitor domains in the anterior endoderm of mouse embryos, using vital dyes to label those cells at 110 somite stage. They identified two distinct types of DE progenitor cells, lateral and medial, arising from three spatially separated embryonic domains. These domains converge to generate the epithelial cells of the liver bud [13]. Intriguingly, the expression of $P y y$ follows a similar pattern as that observed by the lineage tracing of the liver bud progenitors. However, pancreatic progenitors were rarely labeled by this lineage tracing [13] suggesting that $P \gamma y$ may not mark the identical domain. Deletion of $P y y$ in mice does not reveal any obvious defects in endoderm patterning $[46,47]$. However, genetic lineage tracing using Pyy-Cre and a ROSA26 reporter mouse strain demonstrated that in the adult, descendants of Pyy-expressing cells can contribute to the periphery of pancreatic islets and the L-type cells of the distal intestine [46]. The relationship between these later descendants and the early expression patterns has not been explored. Regardless, the dynamic expression pattern of $P y y$ appears to reiterate the morphogenetic movement of foregut progenitors along anterior-posterior and medial-lateral axes prior to tissue specification.

The RIKEN gene, 5730521E12Rik, expressed in the midgut region, is the first known gene that marks exclusively the entire midgut region at early organogenesis stages. Furthermore, 5730521E12Rik is the earliest DE specific and regional marker reported to date. Its early regionalized expression in the few cells in the posterior DE at as early as E7.25 embryo may reflect the early specification of the DE. Tam et al. recently depicted the sequential allocation and global pattern of movement of the DE in the mouse embryo during gastrulation, by tracing cells electroporated with Gfp or painted with carbocyanine dyes [45]. The observations from their study, together with previous fate mapping studies, suggested a probable sequence of allocation of the DE proceeding with (a) the most-posterior endoderm and the dorsal endoderm of the rostral segment of the foregut at early-streak stage; (b) the ventral endoderm of the rostral foregut and additional posterior endoderm at the mid-streak stage; (c) the dorsal and then the ventral endoderm of the posterior segment of the foregut at the late-streak to late-bud stage; and finally, (d) the endoderm of the embryonic mid- and hind-gut at the late-bud to early head-fold stage $[45,48$ 51]. Fascinatingly, the dynamic expression pattern of 5730521E12Rik suggests that it may mark the last population of the DE precursors recruited, thus is possibly a midgut lineage marker. Interestingly, 5730521E12Rik is identical to nephrocan (Nepn), which was recently identified by Mochida et al. as an inhibitor of Transforming Growth Factor- $\beta$ signaling [52]. However, whether $5730521 E 12 R i k$ plays an inhibitory role in vivo during endoderm formation and patterning needs to be further investigated.

The new endoderm genes we identified in this study will provide a valuable tool for further investigation into the underlying molecular mechanisms that regulate endoderm development. In particular, the dynamic expression patterns of $P y y, 5730521$ E12Rik and Trh from E6.5 to E9.5 provide intriguing insights into the endoderm fate mapping studies (Figure 4, 8 and unpublished data). In addition, Cpn1 and 1700011H14Rik showed strikingly similar expression patterns suggesting they may be co-regulated. Further expression and functional analysis of many of these genes will give insights into endoderm development. Moreover, these endoderm genes could be valuable markers to assess and optimize ES cell in vitro differentiation into endoderm and endoderm derivatives.

\section{Conclusion}

We identified novel as well as known genes that are expressed in DE progenitors by analyzing and validating DE longSAGE libraries. These genes provide a valuable tool for further investigation into the molecular mechanisms regulating endoderm development. Our study presents a successful application of analyzing and validating the large amount of data obtained by the Mouse Atlas of Gene Expression Project to identify tissue associated novel genes. The relatively high purity of the tissue source used for the construction of our DE longSAGE libraries and the comparison with a large number of longSAGE libraries from a variety of tissues and embryonic stages are the two critical factors for achieving an efficient screen.

\section{Methods}

\section{Tissue collection and generation of SAGE libraries}

Obtaining enriched DE tissue was achieved by a combination of proteolytic and manual micro-dissection methods [14]. E8.0-E8.5 embryos were isolated from timed pregnant female $\mathrm{C} 57 \mathrm{BL} / 6 \mathrm{~J}$ mice. After removing the extraembryonic membranes, the embryos were transferred to 
$1 \%$ trypsin/Hanks and incubated for 30 minutes on ice. Then $0.01 \%$ DNase $/ 20 \% \mathrm{FBS} /$ Hanks were added to block the activity of trypsin and to digest genomic DNA to reduce stickiness of the tissue. Next, using polished tungsten needles or fine tip forceps, endoderm and ectoderm were separated to minimize mesoderm contamination, and then transferred into Trizol (Invitrogen) (Figure 1A). Somite 0-6 endoderm pieces were pooled for the early whole endoderm library. Somite 8-12 endodermal portions were divided into foregut and hindgut region, and then pooled for foregut and hindgut libraries respectively (Figure 1B). In total, $3.5 \mu \mathrm{g}, 2.7 \mu \mathrm{g}$ and $3.5 \mu \mathrm{g}$ total RNA were isolated from 124 early whole endoderm, 110 foregut and 115 hindgut pieces respectively. RNA quality was assessed using a Bioanalyzer (Agilent). Each SAGE library was constructed with $2.5 \mu \mathrm{g}$ of DNA-free total RNA using the Invitrogen I-SAGE Long kit and protocol as previously described $[32,33]$.

\section{SAGE data analysis}

SAGE data was analyzed using DiscoverySpace software [53]. All SAGE libraries were generated by the Mouse Atlas of Gene Expression project [33]. They were filtered for sequence quality using a 95\% quality cut off for all tags. Tag to gene mapping was performed using the mouse Refseq, MGC and Ensembl databases using the CMOST plugin in DiscoverySpace. Tags were considered sense position matches if they mapped in the same $5^{\prime}$ to 3 ' orientation as the gene, and antisense matches if they mapped in the opposite orientation. Tag 'position' was determined by sequentially numbering NlaIII restriction sites from the 3'-most end (position 1) onward (i.e. next $5^{\prime}$ tag would be position 2, and so on). A tag was considered unambiguous if it mapped to a single gene in a sense position and ambiguous if it mapped to multiple genes in a sense position.

RT-PCR, whole mount in situ hybridization and histology Semi-quantitative RT-PCR followed standard protocols. An ABI 7900 real-time PCR system (Applied Biosystems) and SYBR Green supermix (Applied Biosystems) were used for quantitative real time PCR. RNA from each tissue was prepared using Trizol (Invitrogen). Triplicate cDNAs were obtained by reverse transcription of $1 \mu \mathrm{g}$ of total RNA from newly isolated batches of endoderm tissue. The primers used in the semi-quantitative RT-PCR and realtime PCR are listed in Additional file 3.

Whole mount in situ hybridization was performed as described previously [54]. Probe templates were generated by RT-PCR amplification from total RNAs isolated from E8.0-E8.5 endoderm, with average sizes of 400-800 bp, followed by sequence verification [see Additional file 4]. At least three embryos at each stage were examined for each probe, and restricted expression patterns were con- firmed by independent sets of hybridizations. After whole mount in situ hybridization and photographing, the embryos were embedded by standard procedures in paraffin, sectioned at $8 \mu \mathrm{m}$, dried overnight, dewaxed in xylenes, and mounted for imaging.

\section{Abbreviations}

SAGE: serial analysis of gene expression

DE: definitive endoderm

ES cell: embryonic stem cell

TFs: transcription factors

SPCs: signaling pathway components

\section{Authors' contributions}

$\mathrm{JH}$ conducted most of the experiments and drafted the manuscript. AMC performed the SAGE data analysis. SCL carried out some whole mount in situ hybridization for the validations and proof-read the manuscript. MKW contributed to the design of dissection methods. YZ and MAM supervised the construction of the SAGE libraries. SJMJ supervised the creation of DiscoverySpace software for the SAGE data analysis. PAH conceived of this study, participated in the design of the project, finalized the manuscript and supervised the study. All authors read and approved the final manuscript.

\section{Additional material}

\section{Additional file 1}

Top foregut-specific and hindgut-specific tag sequences. This table shows the top 25 foregut specific and top 20 hindgut specific tag sequences with the counts and gene annotation.

Click here for file

[http://www.biomedcentral.com/content/supplementary/1471213X-7-92-S1.xls]

\section{Additional file 2}

Tag sequences enriched in the endoderm SAGE libraries. This table shows the tag sequences and annotations for transcription factors and signaling pathway components that are enriched in the endoderm with a $T / L$ ration $>0.2$.

Click here for file

[http://www.biomedcentral.com/content/supplementary/1471213X-7-92-S2.xls]

\section{Additional file 3}

Primers used for RT-qPCR. This file contains the primer sequences used for gene validation by quantitative RT-PCR.

Click here for file

[http://www.biomedcentral.com/content/supplementary/1471213X-7-92-S3.pdf] 


\section{Additional file 4}

Primers for amplification of the cDNA fragment for in situ probes. This file contains the primer sequences used to amplify DNA fragments used for in situ hybridization probes.

Click here for file

[http://www.biomedcentral.com/content/supplementary/1471213X-7-92-S4.pdf]

\section{Acknowledgements}

We would like to acknowledge members of the Michael Smith Genome Sciences Centre sequencing, bioinformatics and SAGE library construction platforms for their valuable contributions. Funding for this project was provided by Genome Canada, the British Columbia Cancer Foundation, the National Cancer Institute (USA), and the National Cancer Institute of Canada $(\mathrm{NCIC})$ with funds from the Terry Fox Foundation. M.A.M., S.J.M.J. and P.A.H. are Scholars of the Michael Smith Foundation for Health Research. P.A.H. is a Canadian Institute for Health Research New Investigator.

\section{References}

I. Wells JM, Melton DA: Vertebrate endoderm development. Annu Rev Cell Dev Biol 1999, I 5:393-4I0.

2. Tam PP, Kanai-Azuma M, Kanai Y: Early endoderm development in vertebrates: lineage differentiation and morphogenetic function. Curr Opin Genet Dev 2003, I3(4):393-400.

3. Grapin-Botton A, Melton DA: Endoderm development: from patterning to organogenesis. Trends Genet 2000, 16(3):124-130.

4. D'Amour KA, Agulnick AD, Eliazer S, Kelly OG, Kroon E, Baetge EE: Efficient differentiation of human embryonic stem cells to definitive endoderm. Nat Biotechnol 2005, 23( I 2): I534-I54|.

5. Yasunaga M, Tada S, Torikai-Nishikawa S, Nakano Y, Okada M, Jakt LM, Nishikawa S, Chiba T, Era T, Nishikawa S: Induction and monitoring of definitive and visceral endoderm differentiation of mouse ES cells. Nat Biotechnol 2005, 23(I 2): I542-I550.

6. Lewis SL, Tam PP: Definitive endoderm of the mouse embryo: formation, cell fates, and morphogenetic function. Dev Dyn 2006, 235(9):2315-2329.

7. Fukuda K, Kikuchi Y: Endoderm development in vertebrates: fate mapping, induction and regional specification. Dev Growth Differ 2005, 47(6):343-355.

8. Hart AH, Hartley L, Sourris K, Stadler ES, Li R, Stanley EG, Tam PP, Elefanty $A G$, Robb $L$ : MixII is required for axial mesendoderm morphogenesis and patterning in the murine embryo. Development 2002, I 29(I 5):3597-3608.

9. Kanai-Azuma M, Kanai Y, Gad JM, Tajima Y, Taya C, Kurohmaru M, Sanai Y, Yonekawa H, Yazaki K, Tam PP, Hayashi Y: Depletion of definitive gut endoderm in Sox I7-null mutant mice. Development 2002, I 29( (10):2367-2379.

10. Hoodless PA, Pye M, Chazaud C, Labbe E, Attisano L, Rossant J, Wrana JL: FoxHI (Fast) functions to specify the anterior primitive streak in the mouse. Genes Dev 200I, I 5(10): 1257-I27I.

II. Tremblay KD, Hoodless PA, Bikoff EK, Robertson E): Formation of the definitive endoderm in mouse is a Smad2-dependent process. Development 2000, I27(14):3079-3090.

12. Jung J, Zheng M, Goldfarb M, Zaret KS: Initiation of mammalian liver development from endoderm by fibroblast growth factors. Science 1999, 284(5422): 1998-2003.

13. Tremblay KD, Zaret KS: Distinct populations of endoderm cells converge to generate the embryonic liver bud and ventral foregut tissues. Dev Biol 2005, 280(I):87-99.

14. Wells JM, Melton DA: Early mouse endoderm is patterned by soluble factors from adjacent germ layers. Development 2000 , | 27(8): I $563-1572$.

15. Hebrok M, Kim SK, Melton DA: Notochord repression of endodermal Sonic hedgehog permits pancreas development. Genes Dev 1998, I 2(II): 1705-17/3.

16. Hebrok M, Kim SK, St Jacques B, McMahon AP, Melton DA: Regulation of pancreas development by hedgehog signaling. Development 2000, I 27(22):4905-49|3.
17. Kim SK, Hebrok M, Li E, Oh SP, Schrewe H, Harmon EB, Lee JS, Melton DA: Activin receptor patterning of foregut organogenesis. Genes Dev 2000, I 4(I5): 1866-1871.

18. Kim SK, Hebrok M, Melton DA: Notochord to endoderm signaling is required for pancreas development. Development 1997, I 24(2I):4243-4252.

19. Cleaver O, Krieg PA: Notochord patterning of the endoderm. Dev Biol 200I, 234(I): I-12.

20. Lavon N, Benvenisty N: Study of hepatocyte differentiation using embryonic stem cells. J Cell Biochem 2005, 96(6): $1193-1202$.

21. Hansson M, Tonning A, Frandsen U, Petri A, Rajagopal J, Englund MC Heller RS, Hakansson J, Fleckner J, Skold HN, Melton D, Semb H, Serup P: Artifactual insulin release from differentiated embryonic stem cells. Diabetes 2004, 53( ( 0):2603-2609.

22. Blyszczuk P, Asbrand C, Rozzo A, Kania G, St-Onge L, Rupnik M, Wobus AM: Embryonic stem cells differentiate into insulinproducing cells without selection of nestin-expressing cells. Int J Dev Biol 2004, 48(10): 1095-I 104.

23. Blyszczuk $P$, Wobus $A M$ : Stem cells and pancreatic differentiation in vitro. J Biotechnol 2004, I I 3(1-3):3-13.

24. Loebel DA, Tam PP: A gut feeling. Nat Biotechnol 2005, 23( I 2): $|49|-\mid 492$.

25. Sherwood RI, Jitianu C, Cleaver O, Shaywitz DA, Lamenzo JO, Chen $A E$, Golub TR, Melton DA: Prospective isolation and global gene expression analysis of definitive and visceral endoderm. Dev Biol 2007, 304(2):54I-555.

26. Sinner D, Kirilenko P, Rankin S, Wei E, Howard L, Kofron M, Heasman J, Woodland HR, Zorn AM: Global analysis of the transcriptional network controlling Xenopus endoderm formation. Development 2006, I33(1 0): 1955-1966.

27. Gu G, Wells JM, Dombkowski D, Preffer F, Aronow B, Melton DA: Global expression analysis of gene regulatory pathways during endocrine pancreatic development. Development 2004, I3I(I):165-179.

28. Gitton $Y$, Dahmane N, Baik S, Ruiz i Altaba A, Neidhardt L, Scholze M, Herrmann BG, Kahlem P, Benkahla A, Schrinner S, Yildirimman R, Herwig R, Lehrach $H$, Yaspo ML: A gene expression map of human chromosome 21 orthologues in the mouse. Nature 2002, 420(69 I 5):586-590.

29. Neidhardt L, Gasca S, Wertz K, Obermayr F, Worpenberg S, Lehrach $H$, Herrmann BG: Large-scale screen for genes controlling mammalian embryogenesis, using high-throughput gene expression analysis in mouse embryos. Mech Dev 2000, 98(12):77-94.

30. Wahl MB, Heinzmann U, Imai K: LongSAGE analysis significantly improves genome annotation: identifications of novel genes and alternative transcripts in the mouse. Bioinformatics 2005, 21(8): 1393-1400.

31. Saha S, Sparks AB, Rago C, Akmaev V, Wang CJ, Vogelstein B, Kinzler $\mathrm{KW}$, Velculescu VE: Using the transcriptome to annotate the genome. Nat Biotechnol 2002, 20(5):508-5I2.

32. Khattra J, Delaney AD, Zhao Y, Siddiqui A, Asano J, McDonald H, Pandoh P, Dhalla N, Prabhu AL, Ma K, Lee S, Ally A, Tam A, Sa D, Rogers $S$, Charest D, Stott J, Zuyderduyn S, Varhol R, Eaves C, Jones S, Holt R, Hirst M, Hoodless PA, Marra MA: Large-scale production of SAGE libraries from microdissected tissues, flow-sorted cells, and cell lines. Genome Res 2007, I7(1):108-116.

33. Siddiqui AS, Khattra J, Delaney AD, Zhao Y, Astell C, Asano J Babakaiff R, Barber S, Beland J, Bohacec S, Brown-John M, Chand S, Charest D, Charters AM, Cullum R, Dhalla N, Featherstone R, Gerhard DS, Hoffman B, Holt RA, Hou J, Kuo BY, Lee LL, Lee S, Leung D, Ma K, Matsuo C, Mayo M, McDonald H, Prabhu AL, Pandoh P, Riggins G], de Algara TR, Rupert JL, Smailus D, Stott J, Tsai M, Varhol R, Vrljicak P, Wong D, Wu MK, Xie YY, Yang G, Zhang I, Hirst M, Jones SJ, Helgason CD, Simpson EM, Hoodless PA, Marra MA: A mouse atlas of gene expression: large-scale digital gene-expression profiles from precisely defined developing C57BL/6] mouse tissues and cells. Proc Natl Acad Sci U S A 2005, I 02(5 I): I 8485- I8490.

34. Deutsch G, Jung J, Zheng M, Lora J, Zaret KS: A bipotential precursor population for pancreas and liver within the embryonic endoderm. Development 200I, | 28(6):87|-88|.

35. Ang SL, Wierda A, Wong D, Stevens KA, Cascio S, Rossant J, Zaret $\mathrm{KS}$ : The formation and maintenance of the definitive endoderm lineage in the mouse: involvement of HNF3/forkhead proteins. Development 1993, II9(4): I30I-1315. 
36. Sulik K, Dehart DB, langaki T, Carson JL, Vrablic T, Gesteland K, Schoenwolf GC: Morphogenesis of the murine node and notochordal plate. Dev Dyn 1994, 20 I (3):260-278.

37. Kuo BY, Chen Y, Bohacec S, Johansson O, Wasserman WW, Simpson EM: SAGE2Splice: unmapped SAGE tags reveal novel splice junctions. PLoS Comput Biol 2006, 2(4):e34.

38. Wood HB, Episkopou V: Comparative expression of the mouse SoxI, Sox 2 and Sox 3 genes from pre-gastrulation to early somite stages. Mech Dev 1999, 86(I-2):197-20I.

39. Pieribone VA, Brodin L, Friberg K, Dahlstrand J, Soderberg C, Larhammar D, Hokfelt T: Differential expression of mRNAs for neuropeptide $\mathrm{Y}$-related peptides in rat nervous tissues: possible evolutionary conservation. J Neurosci 1992, | 2(9):336|-337|.

40. Sousa-Nunes R, Rana AA, Kettleborough R, Brickman JM, Clements M, Forrest A, Grimmond S, Avner P, Smith JC, Dunwoodie SL, Beddington RS: Characterizing embryonic gene expression patterns in the mouse using nonredundant sequence-based selection. Genome Res 2003, I3( I 2):2609-2620.

4I. Harrison SM, Dunwoodie SL, Arkell RM, Lehrach H, Beddington RS: Isolation of novel tissue-specific genes from cDNA libraries representing the individual tissue constituents of the gastrulating mouse embryo. Development I995, I 2 I (8):2479-2489.

42. Lu J, Lal A, Merriman B, Nelson S, Riggins G: A comparison of gene expression profiles produced by SAGE, long SAGE, and oligonucleotide chips. Genomics 2004, 84(4):63I-636.

43. Burke Z, Oliver G: ProxI is an early specific marker for the developing liver and pancreas in the mammalian foregut endoderm. Mech Dev 2002, I I 8(I-2): | 47-I55.

44. Thomas PQ, Brown A, Beddington RS: Hex: a homeobox gene revealing peri-implantation asymmetry in the mouse embryo and an early transient marker of endothelial cell precursors. Development 1998, I 25(I):85-94.

45. Tam PP, Khoo PL, Lewis SL, Bildsoe H, Wong N, Tsang TE, Gad JM, Robb L: Sequential allocation and global pattern of movement of the definitive endoderm in the mouse embryo during gastrulation. Development 2007, 134(2):25I-260.

46. Schonhoff S, Baggio L, Ratineau C, Ray SK, Lindner J, Magnuson MA, Drucker DJ, Leiter AB: Energy homeostasis and gastrointestinal endocrine differentiation do not require the anorectic hormone peptide YY. Mol Cell Biol 2005, 25( I0):4189-4I99.

47. Boey D, Lin S, Karl T, Baldock P, Lee N, Enriquez R, Couzens M, Slack K, Dallmann R, Sainsbury A, Herzog H: Peptide YY ablation in mice leads to the development of hyperinsulinaemia and obesity. Diabetologia 2006, 49(6): I360-1370.

48. Lawson KA, Meneses JJ, Pedersen RA: Cell fate and cell lineage in the endoderm of the presomite mouse embryo, studied with an intracellular tracer. Dev Biol I986, I I 5(2):325-339.

49. Lawson KA, Pedersen RA: Cell fate, morphogenetic movement and population kinetics of embryonic endoderm at the time of germ layer formation in the mouse. Development 1987, I0I(3):627-652.

50. Lawson KA, Meneses J], Pedersen RA: Clonal analysis of epiblast fate during germ layer formation in the mouse embryo. Development |99|, I|3(3):89|-9||.

5I. Lawson KA, Pedersen RA: Clonal analysis of cell fate during gastrulation and early neurulation in the mouse. Ciba Found Symp 1992, 165:3-21; discussion 21-6.

52. Mochida Y, Parisuthiman D, Kaku M, Hanai J, Sukhatme VP, Yamauchi $M$ : Nephrocan, a novel member of the small leucine-rich repeat protein family, is an inhibitor of transforming growth factor-beta signaling. | Biol Chem 2006, 28 I (47):36044-3605 I.

53. Robertson N, Oveisi-Fordorei M, Zuyderduyn SD, Varhol RJ, Fjell C, Marra $M$, Jones S, Siddiqui A: DiscoverySpace: an interactive data analysis application. Genome Biol 2007, 8(I):R6.

54. Wilkinson DG, Nieto MA: Detection of messenger RNA by in situ hybridization to tissue sections and whole mounts. Methods Enzymol 1993, 225:361-373.
Publish with Bio Med Central and every scientist can read your work free of charge

"BioMed Central will be the most significant development for disseminating the results of biomedical research in our lifetime. "

Sir Paul Nurse, Cancer Research UK

Your research papers will be:

- available free of charge to the entire biomedical community

- peer reviewed and published immediately upon acceptance

- cited in PubMed and archived on PubMed Central

- yours - you keep the copyright
BioMedcentral 